\title{
Reentrant phase transitions of DNA-surfactant complexes
}

\author{
Rema Krishnaswamy, ${ }^{1}$ V. A. Raghunathan, ${ }^{1}$ and A. K. Sood ${ }^{2}$ \\ ${ }^{1}$ Raman Research Institute, Bangalore 560 080, India \\ ${ }^{2}$ Department of Physics, Indian Institute of Science, Bangalore 560 012, India
}

\begin{abstract}
Complexes of double-stranded DNA with the cationic surfactant cetyltrimethylammonium bromide have been studied using small angle x-ray diffraction at varying concentrations of DNA and the cosurfactant hexanol. At low DNA concentrations, an intercalated hexagonal $\left(H_{I}^{c}\right) \rightarrow$ lamellar $\left(L_{\alpha}^{c}\right) \rightarrow$ inverted hexagonal $\left(H_{I I}^{c}\right)$ transformation is found on increasing hexanol content. The $H_{I I}^{c}$ structure is converted into $L_{\alpha}^{c}$ on adding more DNA. Further increase in hexanol content leads to a phase separation in the surfactant solution, and a reentrant $L_{\alpha}^{c} \rightarrow H_{I I}^{c} \rightarrow L_{\alpha}^{c}$ transition is observed as DNA concentration is increased. Such structural transformations of DNA-surfactant complexes, driven by DNA concentration, have not been reported until now.
\end{abstract}

Complexes of double-stranded (ds) DNA with cationic lipids and surfactants, in aqueous solutions, have been the subject of many experimental and theoretical investigations [1-13]. They form mainly as a result of the entropy gain on the release of counterions that are condensed on DNA and the lipid aggregates $[10,11,14]$. Complexes of ds DNA with double-tailed cationic lipids are found to exhibit either a lamellar $\left(\mathrm{L}_{\alpha}^{c}\right)$ or a two-dimensional (2D) inverted hexagonal $\left(H_{I I}^{c}\right)$ phase depending upon the lipids used [1,3]. Diffraction pattern of the lamellar phase is consistent with an intercalated structure, with the DNA molecules sandwiched between lipid bilayers [Fig. 1(a)]. The hexagonal phase consists of close-packed inverted cylindrical micelles, with the DNA confined in their aqueous cores [Fig. 1(b)]. Recently, we have reported an intercalated hexagonal phase $\left(H_{I}^{c}\right)$ in DNACTAB (cetyltrimethylammonium bromide) complexes, where each DNA molecule is surrounded by three cylindrical micelles [Fig. 1(c)] [7]. The $H_{I}^{c}$ and $L_{\alpha}^{c}$ structures are found in systems where the amphiphilic molecules form cylindrical micelles and bilayers, respectively. On the other hand, the $H_{I I}^{c}$ phase is seen when they form bilayers with very low bending rigidity $\kappa$, due to the more efficient neutralization of DNA by the lipid head groups, possible in such a structure; it is also found in the case of amphiphilic systems with a negative spontaneous curvature [3]. Phase diagrams of cationic lipid-DNA complexes, computed theoretically, show the rather complex phase behavior possible in these systems depending on the bilayer rigidity, charge density, and spontaneous curvature [13].

In this paper we present results of small angle x-ray diffraction studies on DNA-CTAB-hexanol complexes. Our results are summarized in the phase diagram presented in Fig. 2, which shows the structures of the complexes obtained at different hexanol and DNA concentrations. Interestingly, this system displays all the three known equilibrium structures of DNA-lipid/surfactant complexes shown in Fig. 1. The complexes formed at low DNA content exhibit a $H_{I}^{c} \rightarrow L_{\alpha}^{c} \rightarrow H_{I I}^{c}$ transformation on increasing hexanol concentration. The surfactant solution phase separates, when more hexanol is added, and the structure of the complex reverts back to $L_{\alpha}^{c}$. These observations are in accordance with the phase behav- ior of the CTAB-hexanol-water system [15], and the well known reduction in $\kappa$ in the presence of hexanol [16]. However, different sequences of structures are observed as a function of DNA concentration: $H_{I I}^{c} \rightarrow L_{\alpha}^{c}$ at intermediate and $L_{\alpha}^{c} \rightarrow H_{I I}^{c} \rightarrow L_{\alpha}^{c}$ at high hexanol content.

Sodium salt of calf thymus ds DNA (30-50 kbp) was purchased from Sigma. CTAB and hexanol were obtained from Aldrich. The surfactant solutions were prepared in deionized water (Millipore), with the relative hexanol content, $\beta(=[$ hexanol $] /[\mathrm{CTAB}])$, varying from 0 to 10.0 . [CTAB] was fixed at $10 \mathrm{~m} M$. The complexes form immediately on adding DNA to the surfactant solution. They were allowed to equilibrate for about a week and were then transferred to thin-walled glass capillaries of $1 \mathrm{~mm}$ diameter along with some of the supernatant for x-ray diffraction studies. All the diffraction patterns were collected at $30^{\circ} \mathrm{C}$. The relative concentration of DNA, $\rho=$ (weight of CTAB)/ (weight of DNA), was varied over a wide range about the isoelectric point, $\rho_{\text {iso }}(=1.1)$, where the positive charges of the $\mathrm{CTA}^{+}$ions are balanced by the negative charges on DNA. $\mathrm{Cu} K \alpha$ radiation from a rotating anode x-ray generator (Rigaku, UltraX 18) operating at $50 \mathrm{kV}$ and $80 \mathrm{~mA}$ was used to produce the diffraction patterns, which were collected on an image plate (Marresearch). The instrumental resolution (full width at half maximum) was typically $0.2 \mathrm{~nm}^{-1}$.

All the complexes are found to be birefringent under a polarizing microscope, irrespective of hexanol and DNA concentrations. For $\beta<5.0$, diffraction patterns of the complexes show three peaks in the small angle region (Fig. 3, curves $a$ and $b$ ). The magnitudes of their scattering vector $q$ are in the ratio $1: \sqrt{3}: 2$, corresponding to the $(1,0),(1,1)$, and $(2,0)$ reflections from a $2 \mathrm{D}$ hexagonal lattice. We have recently shown that ds DNA-CTAB complexes exhibit an intercalated hexagonal structure shown in Fig. 1(c) [7]. It occurs in amphiphilic systems, such as CTAB, that form cylindrical micelles and has not been considered in any of the theoretical analyses of DNA-lipid complexes reported in the literature. Unlike the case of double-tailed cationic lipids, the area per head group in CTAB is much smaller than the effective area per charge of DNA. Hence these complexes are always overcharged with excess CTAB. We expect this 

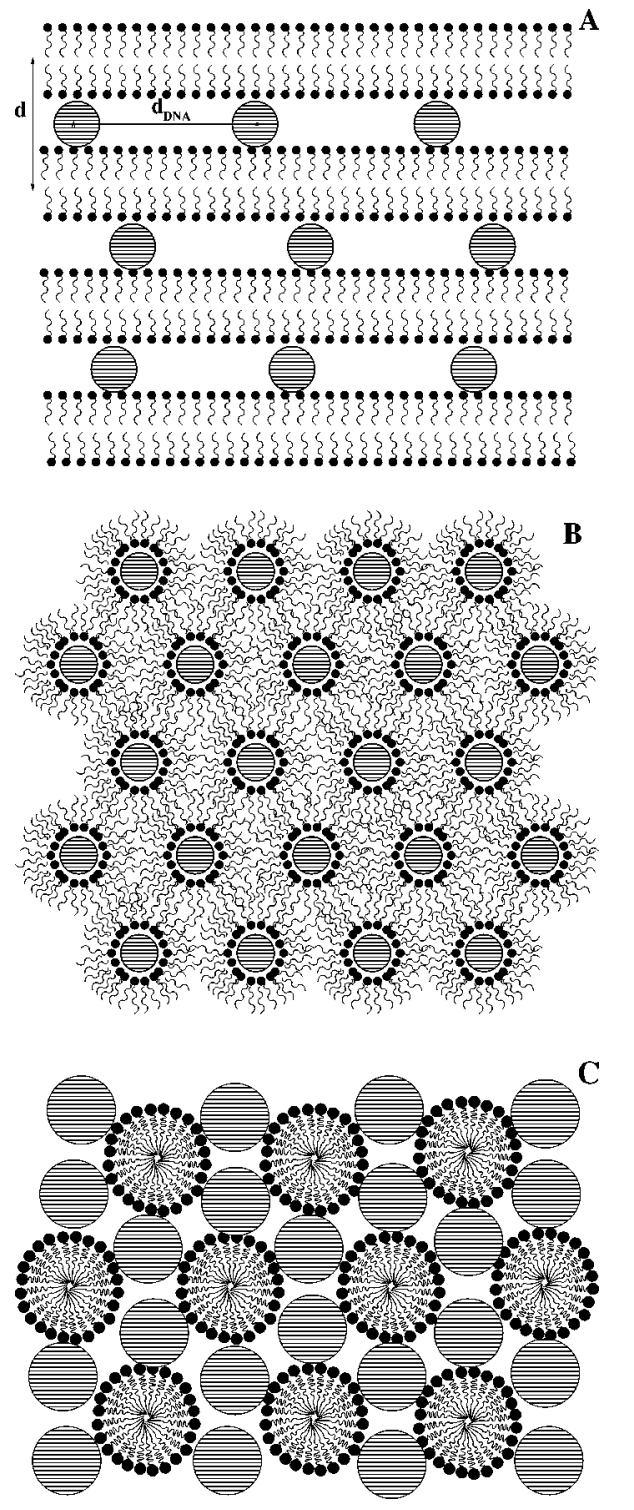

FIG. 1. Schematic diagrams of (a) the lamellar phase $\left(L_{\alpha}^{c}\right)$ of DNA-surfactant complexes, where the DNA strands (denoted by the shaded circles) are sandwiched between surfactant bilayers, (b) the inverted hexagonal phase $\left(H_{I I}^{c}\right)$, where the DNA are confined to the aqueous cores of the micelles, and (c) the intercalated hexagonal phase $\left(H_{I}^{c}\right)$, where each DNA strand is surrounded by three cylindrical micelles.

structure to persist in the presence of small amounts of hexanol. Since hexanol molecules are not confined to the micelle-water interface, the surface charge density of the micelles cannot be directly estimated, although it can be expected to decrease with hexanol content. At $\beta=0$, the lattice parameter $a=5.64 \pm 0.05 \mathrm{~nm}$, and is insensitive to the DNA concentration. It reduces to $5.50 \pm 0.05 \mathrm{~nm}$ at $\beta=3.5$ for low DNA concentration $(\rho=36.0)$, indicating the gradual thinning of the cylindrical micelles on adding hexanol. Further, at this hexanol concentration, $a$ is lower at high DNA concentration, being $5.23 \pm 0.05 \mathrm{~nm}$ at $\rho=1.0$. This probably arises from the thinning of the cylindrical micelles in order to incorporate larger amounts of DNA within the complex.

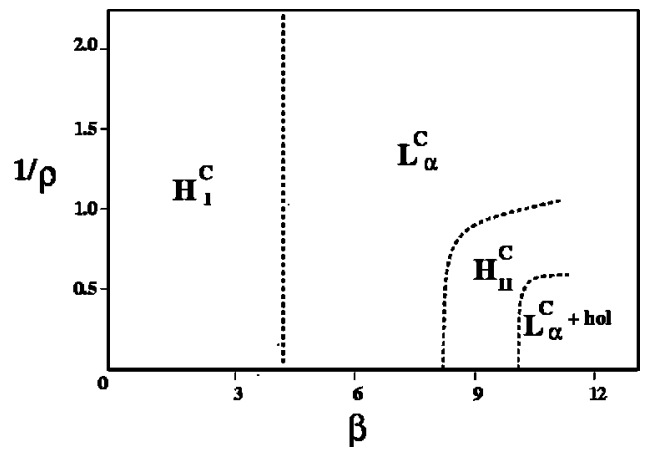

FIG. 2. The phase diagram of the system at $30^{\circ} \mathrm{C}$, showing the different complexes obtained as a function of hexanol and DNA concentrations. $\beta=[$ hexanol $] /[\mathrm{CTAB}], \quad \rho=($ weight of $\mathrm{CTAB}) /$ (weight of DNA). hol denotes the hexanol rich phase coexisting with the complex. The locations of the different phase boundaries have not been precisely determined.

This behavior is similar to the uptake of excess DNA seen in lamellar DNA-lipid complexes leading to overcharging, and is caused by the higher free energy of uncomplexed DNA in solution [6]. The hexanol free micelles are more rigid and hence can be expected to resist such deformations.

Structure of the complexes changes from hexagonal to lamellar on increasing hexanol concentration, at $\beta \sim 5.0$. Diffraction patterns of this phase usually have two to three peaks in the small angle region, arising from the lamellar structure (Fig. 3 curves $c$ and $d$ ), which give a lamellar periodicity comparable to the sum of the bilayer thickness and the diameter of a hydrated DNA. In addition it also shows a peak, whose position depends significantly on DNA concentration near the isoelectric point. This behavior is very similar to that seen in lamellar DNA-lipid complexes $[1,6]$. Hence the lamellar complexes under study can also be presumed to have a similar structure, with the additional diffraction peak arising from the average lateral separation $d_{D N A}$ between DNA strands [Fig. 1(a)]. This peak is identified by an arrow in all the diffraction patterns of this phase presented

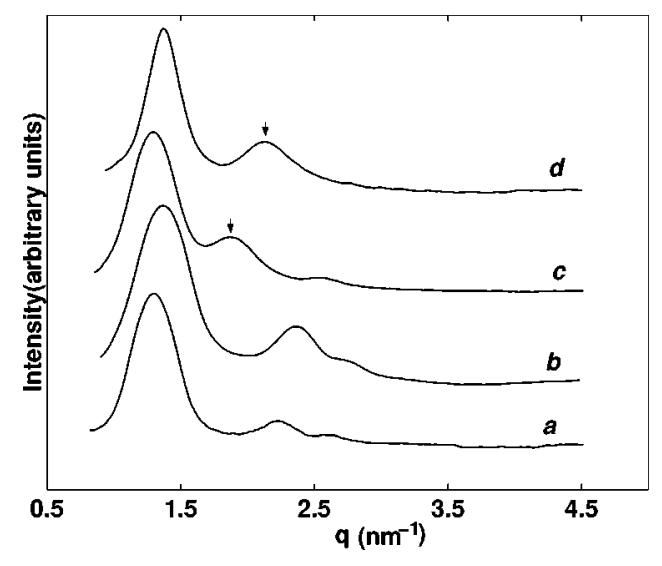

FIG. 3. Diffraction patterns of DNA-CTAB-hexanol complexes in the $H_{I}^{c}$ (curves $a$ and $b$ ) and $L_{\alpha}^{c}$ (curves $c$ and $d$ ) phases at $\beta=3.5$ and 5.0, respectively. Curves $a$ and $c$ correspond to $\rho=36.0$ and $b$ and $d$ to $\rho=1.0$. The arrows on curves $c$ and $d$ indicate in-plane DNA-DNA correlation peak. 


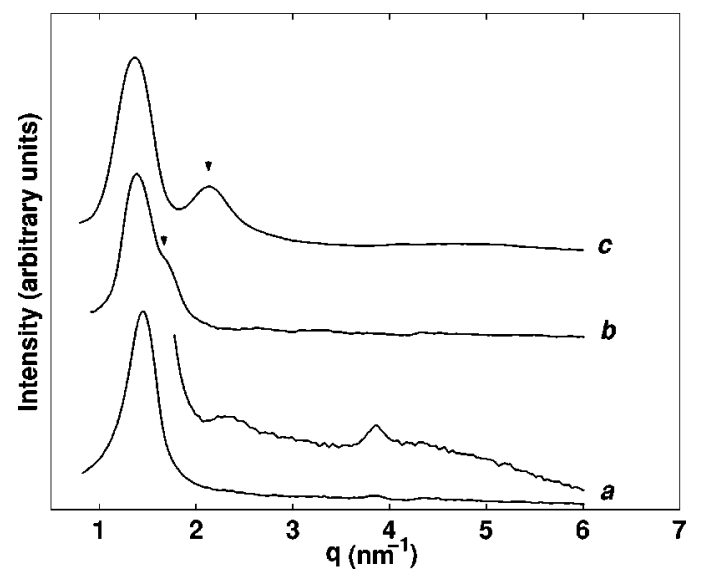

FIG. 4. Diffraction patterns of DNA-CTAB-hexanol complexes in the $H_{I I}^{c}$ (curve $a$ ) and $L_{\alpha}^{c}$ (curves $b$ and $c$ ) phases at $\beta=9.0$. The three curves correspond to $\rho=36.0$ (curve $a$ ), 1.0 (curve $b$ ), and 0.9 (curve $c$ ). The peak at $q=3.85 \mathrm{~nm}^{-1}$ in curve $a$ corresponds to the $(2,1)$ reflection from the $2 \mathrm{D}$ hexagonal lattice, whereas the broad peak at $\sim 2.5 \mathrm{~nm}^{-1}$ can be attributed to the helical structure of DNA. These peaks could be observed clearly only on using an incident beam with a larger cross section. Note the absence of the $(1,1)$ and $(2,0)$ reflections in curve $a$, which are very prominent in the $H_{I}^{c}$ phase (Fig. 3, curves $a$ and $b$ ).

here. High resolution experiments show that the DNA correlation peak is much broader than the lamellar peaks and has a different shape [4]. These differences are, however, not apparent in the present data due to the lower instrumental resolution. The $H_{I}^{c} \rightarrow L_{\alpha}^{c}$ transition of the complexes is consistent with the reported formation of bilayers in the CTABhexanol-water system [15]. The lamellar periodicity $d$ decreases slightly with hexanol concentration from $4.87 \pm 0.05$ $\mathrm{nm}$ at $\beta=5.0$ to $4.70 \pm 0.05 \mathrm{~nm}$ at $\beta=8.0$, due to the thinning of bilayers on adding hexanol. On the other hand, $d_{\mathrm{DNA}}$ at $\rho>\rho_{\text {iso }}$ increases from $3.32 \pm 0.05 \mathrm{~nm}$ to $3.65 \pm 0.05 \mathrm{~nm}$ over this range of $\beta$, due to the gradual decrease in the bilayer charge density.

Further increase in hexanol concentration leads to another structural change above $\beta \sim 8.5$ at low DNA concentrations. The morphology of these complexes is very different from those obtained at lower hexanol concentrations. They separate out from the aqueous solution as a dense precipitate, suggesting their hydrophobic nature, unlike the latter, which remain fairly dispersed in solution. Diffraction patterns of these complexes show only two reflections, one strong and one very weak (Fig. 4, curve $a$ ). Additional reflections were not observed even after exposures lasting many hours. Note that these patterns are very different from those of the $L_{\alpha}^{c}$ and $H_{I}^{c}$ phases (Fig. 3). As mentioned earlier, in addition to these two, a hexagonal structure has also been seen in DNA-lipid complexes in the presence of hexanol [3], consisting of inverted cylindrical micelles with the DNA confined in their aqueous cores [Fig. 1(b)], whose diameter is comparable to that of DNA $(\sim 2.5 \mathrm{~nm})$. The $H_{I I}^{c}$ phase is expected to be favored over $L_{\alpha}^{c}$ in lipids with very flexible bilayers at low charge density [13]. Both these conditions are met on increasing the hexanol concentration in the present system.
Further, since hexanol is not confined to the bilayer water interface, it can preferentially occupy the interstitial regions in the $H_{I I}^{c}$ structure, where three inverted cylindrical micelles meet. This can drastically reduce the frustration of the surfactant chains, which would have been otherwise stretched in order to occupy these regions, and thus stabilize this phase further. The two peaks in (Fig. 4, curve $a$ ) occur at values of $q$, which are in the ratio $1: \sqrt{7}$. On the basis of the above arguments, we tentatively identify this phase as $H_{I I}^{c}$, and index the two reflections as $(1,0)$ and $(2,1)$, with $a$ $=4.99 \mathrm{~nm}$. The hydrophobic nature of the complexes, suggested by the precipitate morphology, is consistent with such a structure. It is also supported by a simple analysis of the diffraction data. The absence of the $(1,1)$ and $(2,0)$ reflections in the diffraction patterns of this complex can be attributed to the fact that the form factor of the electron rich cylindrical core of the micelles has a zero in between the corresponding values of $q$. This gives the radius of the core to be about 1.4 $\mathrm{nm}$, which is very close to that of a hydrated DNA $(\sim 1.25$ $\mathrm{nm})$. Thus all the observations indicate that this phase could indeed be $H_{I I}^{c}$.

The $H_{I I}^{c}$ structure obtained for $8.5 \leqslant \beta \leqslant 9.0$ is transformed into $L_{\alpha}^{c}$ on increasing DNA concentration (Fig. 4, curves $b$ and $c$ ). The distance between two adjacent DNA strands is fixed at the lattice parameter $a$ in the $H_{I I}^{c}$ structure. On the other hand, no such restriction exists in the $L_{\alpha}^{c}$ structure, and $d_{\text {DNA }}$ changes significantly across the isoelectric point. The value of $\rho$ in these two phases, at the same surfactant composition, can be estimated from their lattice parameters. The ratio, $\rho_{H_{I I}} / \rho_{L}=\left(\sqrt{3} a^{2}-2 \pi R^{2}\right) /\left(2 d_{B} d_{D N A}\right)$, where $d_{B}$ is the bilayer thickness and $R$ the radius of a hydrated DNA. Putting $a=5.0 \mathrm{~nm}, R=1.25 \mathrm{~nm}$, and $d_{B}=2.2 \mathrm{~nm}$, this ratio turns out to be $\sim 7.5 / d_{\mathrm{DNA}}$. In the lamellar complexes obtained for $5.0 \leqslant \beta \leqslant 8.0, d_{\text {DNA }}$ is about $3.5 \mathrm{~nm}$ even at low DNA concentrations. Thus it is clear that more DNA can be accommodated in the $L_{\alpha}^{c}$ phase compared to $H_{I I}^{c}$. Whereas at low DNA concentrations the $H_{I I}^{c}$ phase is stabilized by the efficient neutralization of DNA, it becomes unstable in the presence of excess uncomplexed DNA and transforms into $L_{\alpha}^{c}$. Such transformations of the structure of the complexes, driven by DNA concentration, have not been reported in the literature.

The DNA concentration at the $H_{I I}^{c} \rightarrow L_{\alpha}^{c}$ transition should correspond to the maximum amount of DNA that can be incorporated in the former structure. It can be estimated from the geometry of the system and is given by $\rho_{c}=\left(\sqrt{3} a^{2}\right.$ $\left.-2 \pi R^{2}\right) \varrho_{s} f /\left(2 A_{D} \varrho_{D}\right)$, where $a$ is the lattice parameter, $R$ the radius of the aqueous core $(1.25 \mathrm{~nm}), \varrho_{s}$ the surfactant density, $f$ the weight fraction of CTAB in the micelle, $A_{D}$ the average area of DNA (molar volume/contour length, $\left.1.86 \mathrm{~nm}^{2}\right)$, and $\varrho_{D}$ the density of DNA $(1.7 \mathrm{~g} / \mathrm{cc})$. At $\beta=9.0$, $\rho_{c}$ turns out to be $\sim 1.3$, which is very close to the experimental value of $\sim 1.0$.

As mentioned earlier, for $\beta>10.0$, the surfactant solution phase separates, with the formation of a hexanol rich phase [15]. At low DNA concentrations, the complex formed coexists with the hexanol rich phase. Diffraction pattern of this 


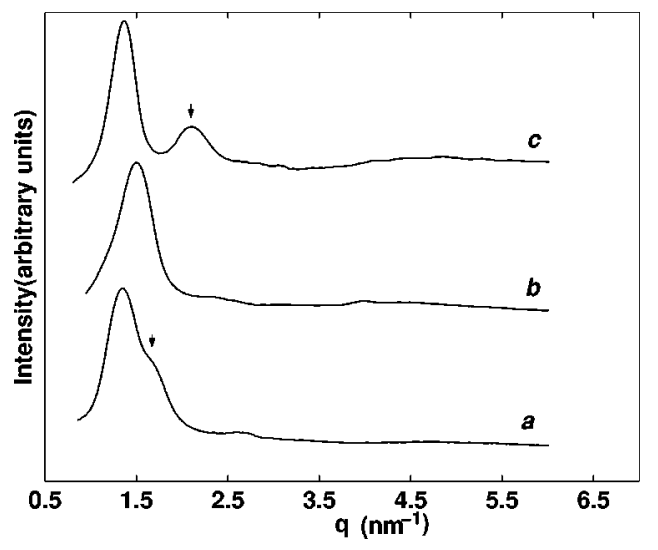

FIG. 5. Diffraction patterns of DNA-CTAB-hexanol complexes at $\beta=10.0$. The $L_{\alpha}^{c}$ phase at $\rho=36.0$ (curve $a$ ) is converted into $H_{I I}^{c}$ at $\rho=1.0$ (curve $b$ ) and back into $L_{\alpha}^{c}$ at $\rho=0.5$ (curve $c$ ). Note the shift of the DNA correlation peak to higher $q$ in (curve $c$ ) compared to (curve $a$ ), due to the incorporation of higher amounts of DNA in the complex.

complex corresponds to the $L_{\alpha}^{c}$ phase, with a $d_{\mathrm{DNA}}$ of $\sim 3.7$ $\mathrm{nm}$ (Fig. 5, curve $a$ ). The formation of this phase indicates that the hexanol concentration in the vesicular phase is less than that in the solution just before phase separation. We have not determined the composition of the two phases at $\beta=10$, but the fact that $d_{\mathrm{DNA}}$ in this case is very close to that obtained at $\beta=8$, at low DNA concentration, supports this conclusion. On increasing DNA concentration, the hexanol rich phase gradually disappears. This increases the hexanol content in the complex and its structure becomes $H_{I I}^{c}$ (Fig. 5, curve $b$ ), presumably due to the decrease in both $\kappa$ and the bilayer charge density. Like the $H_{I I}^{c}$ structure obtained for $8.5 \leqslant \beta \leqslant 9.0$, this structure also transforms into the $L_{\alpha}^{c}$ phase on further addition of DNA, with $d_{\mathrm{DNA}}=2.96 \pm 0.05 \mathrm{~nm}$ (Fig. 5, curve $c$ ). The fact that $d_{\text {DNA }}$ in this case is much less than that in the $L_{\alpha}^{c}$ phase obtained at very low DNA concentrations is consistent with the $H_{I I}^{c} \rightarrow L_{\alpha}^{c}$ transition being

[1] J.O. Rädler, I. Koltover, T. Salditt, and C.R. Safinya, Science 275, 810 (1997).

[2] T. Salditt, I. Koltover, J.O. Rädler, and C.R. Safinya, Phys. Rev. Lett. 79, 2582 (1997).

[3] I. Koltover, T. Salditt, J.O. Rädler, and C.R. Safinya, Science 281, 78 (1998).

[4] T. Salditt, I. Koltover, J.O. Rädler, and C.R. Safinya, Phys. Rev. E 58, 889 (1998).

[5] F. Artzner, R. Zantl, G. Rapp, and J.O. Rädler, Phys. Rev. Lett. 81, 5015 (1998).

[6] I. Koltover, T. Salditt, and C.R. Safinya, Biophys. J. 77, 915 (1999).

[7] R. Krishnaswamy, P. Mitra, V.A. Raghunathan, and A.K. Sood, Europhys. Lett. 62, 357 (2003).

[8] C.S. O'Hern and T.C. Lubensky, Phys. Rev. Lett. 80, 4345 driven by the denser packing of DNA in the latter. Further, this transition is found to occur in a narrow range of $0.5<\rho$ $<1.0$, in good agreement with the estimated $\rho_{c} \sim 1.0$. These structural transformations of the complexes are also accompanied by the corresponding morphological changes of the precipitates, described earlier.

The phase behavior of DNA-lipid complexes has been theoretically investigated, based on the Poisson-Boltzmann equation for the counterion distribution, and the Helfrich Hamiltonian for the bilayer deformations [13]. These calculations find a $L_{\alpha}^{c} \rightarrow H_{I I}^{c}$ transition in very flexible bilayers at low charge density. The observation of the $H_{I I}^{c}$ structure at high hexanol concentration is in qualitative agreement with these predictions. A quantitative comparison with the predicted phase behavior cannot, however, be made, since the cosurfactant molecules are not confined to the water-bilayer interface, unlike the neutral lipids considered in the theory. The addition of hexanol leads to simultaneous lowering of the charge density and bare rigidity of the bilayers, whereas the former can, in principle, be independently decreased by adding a neutral lipid.

In conclusion, we have studied complexes formed by ds DNA with the single-tailed cationic surfactant CTAB, in the presence of the cosurfactant hexanol. At low DNA concentrations, the complexes exhibit a $H_{I}^{c} \rightarrow L_{\alpha}^{c} \rightarrow H_{I I}^{c}$ transition on increasing the hexanol content. This trend is in accordance with the known influence of hexanol on the structure and properties of CTAB aggregates. A $H_{I I}^{c} \rightarrow L_{\alpha}^{c}$ transformation is observed as a function of DNA concentration at higher hexanol concentrations, which can be understood in terms of the higher DNA/surfactant ratio in the latter structure. We hope that our results will motivate further theoretical work to understand the rich phase behavior exhibited by DNAsurfactant-cosurfactant systems.

We thank Yashodhan Hatwalne and Madan Rao for helpful discussions.

(1998)

[9] L. Golubović and M. Golubović, Phys. Rev. Lett. 80, 4341 (1998).

[10] R. Bruinsma, Eur. Phys. J. B 4, 75 (1998).

[11] D. Harries, S. May, W.M. Gelbart, and A. Ben-Shaul, Biophys. J. 75, 159 (1998).

[12] N. Dan, Biophys. J. 73, 1842 (1997).

[13] S. May, D. Harries, and A. Ben-Shaul, Biophys. J. 78, 1681 (2000).

[14] F. Oosawa, J. Polym. Sci. 23, 421 (1957); G.S. Manning, J. Chem. Phys. 51, 924 (1969).

[15] P. Ekwall, L. Mandell, and K. Fontell, J. Colloid Interface Sci. 29, 639 (1969).

[16] C.R. Safinya, E.B. Sirota, D. Roux, and G.S. Smith, Phys. Rev. Lett. 62, 1134 (1989). 